\title{
A proposito di un caso di ricostruzione della mano mediante uso di sostituto dermico
}

A. Marchesi, M. Brioschi, E. Benanti, S.Marcelli, L.Vaienti

Dipartimento di Chirurgia Plastica-Ricostruttiva, I.R.C.C.S Policlinico San Donato-Università degli Studi di Milano, San Donato Milanese (MI)

ABSTRACT About a case of reconstruction of the hand by the use of a dermal substitute

Soft-tissues defects of the hand still represent a surgical challenge for plastic surgeons, due to paucity of local tissues, especially in case of post-traumatic extended wounds. Up to now, many surgical reconstructions have been proposed, such as skin grafts, local o free flaps and dermal substitutes. We present the case of large defect of the hand, reconstructed with a der mal substitute based on a hyaluronan acid scaffold. The result was satisfactory and the patient healed in 30 days.

At one-year follow-up the patient presented minimum scar retractions with a good functional and aesthetic outcome.

\section{Introduzione}

Il trattamento di ferite complesse con esposizione di tessuti profondi, quali tendini, muscoli, vasi e nervi, da sempre rappresenta una sfida ricostruttiva nell'ambito della chirurgia della mano.

Il ripristino di una copertura tegumentaria adeguata a livello della mano risulta di capitale importanza. Infatti, nonostante essa rappresenti solo il 3\% dell'intera superficie corporea, come riconosciuto dalla American Burn Association, la perdita di una mano comporta un danno di circa il $57 \%$ in termini di funzione dell'intera persona [1].

Negli ultimi quattro decenni sono state descritte numerose tecniche, tra le quali innesti dermo-epidermici, lembi locali o lembi liberi, nonché l'uso di sostituti dermici. Riportiamo il caso di un paziente trattato con l'uso di un sostituto dermico in combinazione con tecniche chirurgiche tradizionali, per il trattamento di una ferita complessa a livello della mano.

\section{Caso clinico}

Donna di 35 anni che, in seguito a trauma accidentale da schiacciamento, riportò ampia perdita di sostanza dei tessuti molli e di due dita, a livello della mano sinistra (Fig. 1). La paziente non presentava comorbilità di rilievo e l'anamnesi fisiologica era negativa per potus e tabagismo.

La paziente, ricoverata dapprima presso altra struttura, venne sottoposta a intervento di asportazione di IV e V dito e chiusura per prima intenzione dei lembi della ferita. Alla nostra osservazione, a 6 giorni dal trauma, la paziente presentava ampia necrosi dei tessuti molli sul versante ulnare del palmo. Venne quindi sottoposta a intervento urgente di pulizia chirurgica della ferita con rimozione di tutti i tessuti necrotici e, ovviamente, tamponi della ferita a scopo colturale. Il bilancio lesionale fu quello di esposizione os sea a livello del $\mathrm{V}$ metacarpo per un tratto di $2 \mathrm{~cm}$ di lunghezza e perdita dei tessuti molli dell'intero lato ulnare della mano (Fig. 2). Considerato l'elevato rischio di infezione della ferita, si decise di

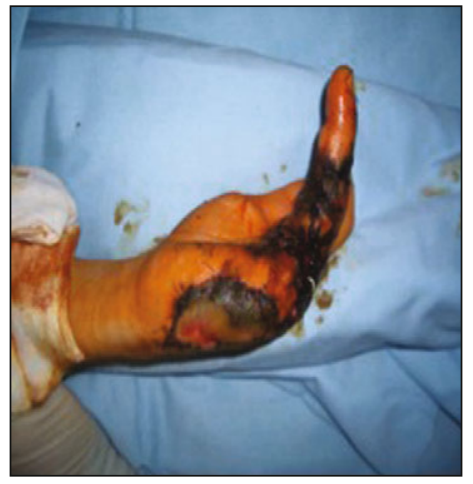

Fig. 1.Condizione preoperatoria con ampia

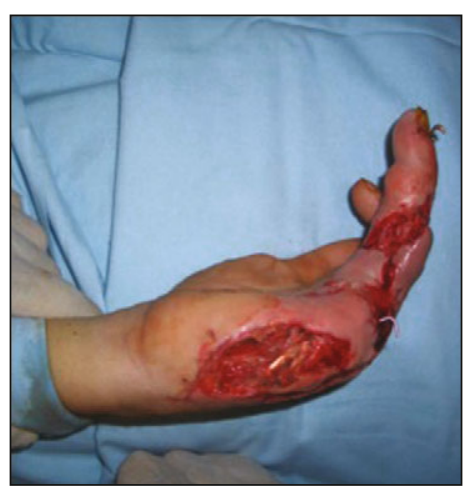

Fig. 2. Condizione dopo prima toilette chirurgica con esposizione ossea del V metacarpo

effettuarne la copertura per 14 giorni con sostituto dermico basato su uno scheletro di acido ialuronico (Hyalomatrix ${ }^{\circledR}$ ), con lo scopo di stimolare i processi di guarigione e di granulazione, in attesa dell'esito dei tamponi colturali (Fig. 3); una volta debellata l'infezione locale attraverso terapia antibiotica mirata con levofloxacina $500 \mathrm{mg}$ x 2/die per 10 giorni, si scelse di evitare un inervento di copertura chirurgica mediante innesto dermo-epidermico in quanto la lesione presentava un ottimo tessuto di granulazione, che a 15 giorni portò a completa guarigione della ferita con riepitelizzazione spontanea (Fig 4). Il controllo a 1 anno mocace, senza episodi successivi di ulcerazione.

\section{Discussione}

La ricostruzione dei tessuti molli della mano costituisce da sempre un tema altamente discusso. $\mathrm{Ne}$ caso di ampie ferite comportanti l'esposizione delle cosiddette "strutture nobili profonde", il soescara e perdita di IV eV dito strò una copertura cutanea effi- lo uso di un innesto dermo-epidermico quasi sempre comporta una copertura inadeguata sia da punto di vista anatomico sia da quello funzionale. Pertanto, tradizionalmente in questo tipo di ferite il trattamento di prima scelta è rappresentato dalla copertura mediante lembi, locali o liberi. Riguardo alle unità ricostruttive locali, sono stati descritti il lembo antibrachiale radiale a peduncolo

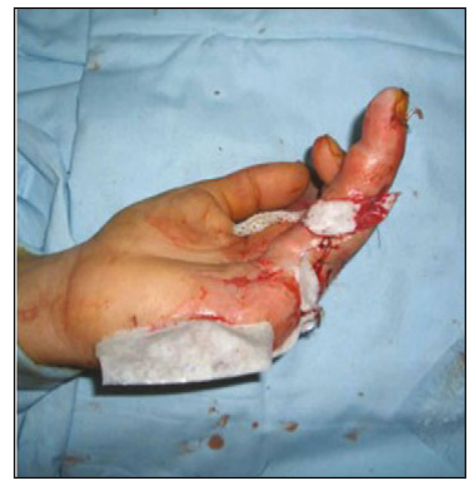

Fig. 3. Applicazione di sostituto dermico (Hyalomatrix)

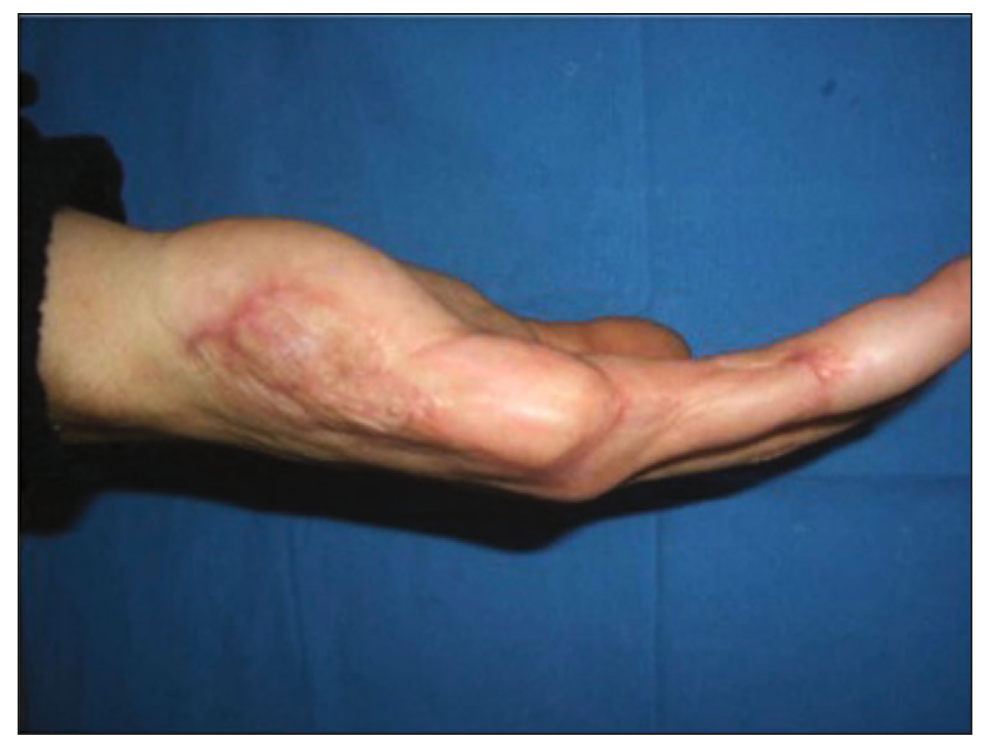

Fig. 4. Risultato post-operatorio a 30 giorni

distale [2] o il lembo interosseo posteriore [3]. Per quanto concerne invece le possibili risorse tissutali prelevate a distanza, si possono annoverare il lembo brachiale mediale [4] e quello brachiale laterale [5]; i lembi inguinale [6], peroneale [7] e parascapolare [8], indicati per ampie perdite di sostanza, hanno spesso lo svantaggio di essere troppo grossi e pertanto necessitano di ulteriori interventi di rimodellamento. Anche se tali lembi spesso of frono un'adeguata copertura di tendini e ossa, spesso gli svantaggi consistono in importanti menomazioni a carico delle sedi donatrici, quali il sacrificio dell'arteria radiale nel caso del lembo antibrachiale, oltre che tempi operatori prolungati e difficoltà tecniche nella dissezione chirurgica. Attualmente l'evoluzione scientifica nel campo della chirurgia pla stica ha spostato sempre più l'attenzione da un approccio pura-

mente ricostruttivo a un approc cio rigenerativo, attraverso l'introduzione di numerosi materiali bio-ingegnerizzati. Nel campo della chirurgia della mano hanno trovato utile impiego per la ricostruzione immediata o differita dei tessuti tegumentari alcuni di questi prodotti, basati su collagene $[9,10]$ o su acido ialuronico [11]

In questo articolo riportiamo la nostra esperienza nell'uso di un sostituto dermico composto da uno strato di acido ialuronico esterificato coperto da una membrana di silicone. Le fibre di silicone, a contatto diretto con il fondo della ferita, vengono gradualmente assorbite e nel contempo stimolano i fibroblasti in una ordinata deposizione dei componenti della neomatrice extracellulare. Lo strato esterno di silicone, nel frattempo, protegge la ferita da possibili contaminazioni da permettere la copertura di picture nobili profonde. Il risultato a distanza ha mostrato una buona copertura cutanea sia dal punto di vista funzionale sia da quello estetico, evitando il sacrificio di sedi donatrici.I

Bibliografia

ccauley RL (2009) Reconstruction of the pediatric bur-
D01 10.1007/s10261-013-0062-1

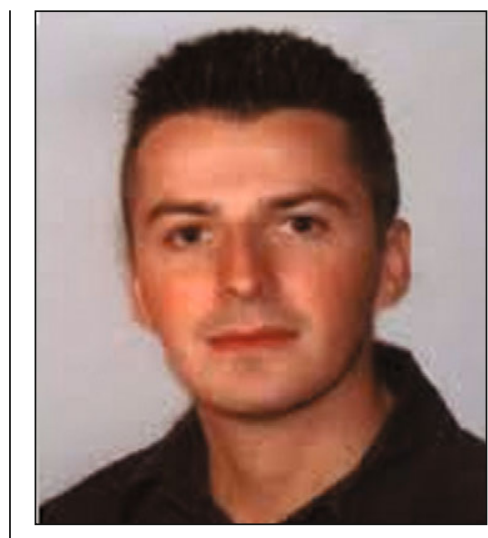

A. Marchesi

ned hand. Hand Clin 25:543550

Foucher G, van Genechten F Merle N et al (1984) A compound radial artery forearm flap in hand surgery: an original modification of the Chinese forearm flap. Br J Plast Surg 37:139-148

Neuwirth M, Hubmer M, Koch H (2013) The posterior interosseous artery flap: clinical results with special emphasis on donor site morbidity. J Plast Reconstr Aesthet Surg 66:623-628 Bhattacharya S, Bhagia SP, Bhatnagar SK et al (1991) The medial upper arm flap. An alternative flap to cover palmar defects of hand and distal forearm. J Hand Surg Br 16:342-345

Moffett TR, Madison SA, Derr JW Jr et al (1992) An extended approach for the vascular pedicle of the lateral arm free flap. Plast Reconstr Surg 89:259-267 Chow JA, Bilos ZJ, Hui P et al (1986) The groin flap in reparative surgery of the hand. Plast Reconstr Surg 77:421-426

Nakashima H, Araki Y, Nishikido E (1987) Free peroneal flap for wide skin defects of the foot and volar scar contracture of the hand. J Reconstr Microsurg 3:105-111

Burns JT Schlafly B (1986) Use of the parascapular flap in hand reconstruction. J Hand Surg Am 11:872-875

Cuadra A Correa G Roa Ret a (2012) Functional results of burned hands treated with Integra ${ }^{\circledR}$. Plast Reconstr Aesthet Surg 65:228-234

10. Ryssel H, Germann G, Kloeters $O$ et al (2010) Dermal substitution with Matriderm( $(\circledR)$ in burns on the dorsum of the hand. Burns $36 \cdot 1248-1253$

11. Vaienti L, Marchesi A, Palitta G et al (2013) Limb trauma: the use of an advanced wound care device in the treatment of full-thickness wounds. Strategies Trauma Limb Reconstr 8:111-115

12. West DC, Hampson IN, Arnold F et al (1991) Angiogenesis induced by degradation products of hyaluronic acid. Science 228 . 1324-1326
Lovvorn HN, Cass DL, Sylvester KG et al (1998) Hyaluronan receptor expression increases in fetal excisional skin wounds and correlates with fibroplasia. J Pe-

Croce MA, Dyne K, Boraldi F et al (2001) Hyaluronan affects protein and collagen synthesis by in vitro human skin fibroblasts. Tissue Cell 33:326-331 diatr Surg 33:1062-1069 